\title{
Transformations of Germs of Differentiable Functions through Changes of Local Coordinates
}

\author{
By
}

\author{
Masahiro Shiota
}

\section{$\S 1$. Introduction}

We generalize the results in N. Levinson [3], [4] and J. Cl. Tougeron [8] which show that some germs of differentiable (or analytic) functions are transformed through changes of coordinates into polynomials in one (or two) variable with coefficients which are germs in the other variables (§3).

H. Whitney [9] has shown that $x y(y-x)(y-(3+t) x)(y-\gamma(t) x)$ (where $\gamma$ is a transcendental function and $\gamma(0)=4$ ) cannot be transformed into any polynomial through analytic changes of coordinates (locally at the origin), and we prove this function cannot be transformed even through differentiable changes of coordinates $(\$ 4)$.

In view of Thom's Principle, that is, for a germ of an analytic function $f$ having 0 as a topologically isolated singularity, the variety $f^{-1}(0)$ determines the function $f$, we study particularly whether a germ $f \phi$ (such that $\phi(0)>0)$ can be transformed into $f(\S 5)$. As a corollary of the sequence we obtain a sufficient condition for a germ of a differentiable function in two variables to be transformed into a germ of an analytic function $(\S 6)$.

In Appendix we show canonical forms of germs which have un point de naissance or un point critique du type queue d'aronde which is due to Cerf [2].

The method of proofs is to use almost all theorems in Malgrange [6]. The author thanks Professor Adachi for his criticism.

\section{§. Definitions}

According to Malgrange [6] we denote respectively by $\mathcal{O}_{n}($ or $\mathcal{O}(x)$ ), Communicated by S. Matsuura, October 4, 1972. 
$\mathscr{E}_{n}$ (or $\mathscr{E}(x)$ ) the rings of germs at 0 in $\mathbf{R}^{n}$ of real analytic and $C^{\infty}$-functions, and by $\mathscr{F}_{n}$ (or $\mathscr{F}(x)$ ) the ring of formal power series in $n$ indeterminates over $\mathbf{R}$. One has a mapping $T: \mathscr{E}_{n} \rightarrow \mathscr{F}_{n}$ (Taylor expansion at 0 ). We regard $\mathcal{O}_{n} \subset \mathscr{F}_{n}$. We denote by $\mathfrak{m}\left(\mathcal{O}_{n}\right)$ (resp. $\mathfrak{m}\left(\mathscr{E}_{n}\right)$ ) the maximal ideal of $\mathcal{O}_{n}$ (resp. $\mathscr{E}_{n}$ ). As $\mathcal{O}_{n}$ and $\mathscr{F}_{n}$ are unique factorization rings, for any $f \in \mathcal{O}_{n}$ (resp. $\left.\in \mathscr{E}_{n}\right) f$ (resp. $T f$ ) can be factorized. So $\mathscr{O}_{n}^{\circ}$ (resp. $\mathscr{E}_{n}^{\circ}$ ) denotes the set of all germs $f$ in $\mathscr{O}_{n}$ (resp. $\mathscr{E}_{n}$ ) such that $f$ (resp. $T f$ ) is not 0 , is in $\mathfrak{m}\left(\mathcal{O}_{n}\right)$ (resp. $\mathfrak{m}\left(\mathscr{E}_{n}\right)$ ) and has no multiple factors. Adif ${ }_{n}$ (resp. Dif $_{n}$ ) denotes the set of analytic local diffeomorphisms (resp. local diffeomorphisms of $C^{\infty}$-class) around 0 in $\mathbf{R}^{n}$. We have

(1) for any $\tau \in \operatorname{Adif}_{n}\left(\right.$ resp. $\left.\operatorname{Dif}_{n}\right)$ and $f \in \mathcal{O}_{n}^{\circ}\left(\right.$ resp. $\left.\mathscr{E}_{n}^{\circ}\right) f \circ \tau \in \mathcal{O}_{n}^{\circ}\left(\right.$ resp. $\left.\mathscr{E}_{n}^{\circ}\right)$,

(2) $\mathcal{O}_{n} \cap \mathscr{E}_{n}^{\circ}=\mathcal{O}_{n}^{\circ} \quad$ (Zariski-Nagata).

For any analytic set $F$ in $\Omega$ (where $\Omega \ni a, \Omega$ open in $\mathbf{R}^{n}$ ), the germ of $F$ at $a$ is called an analytic germ at $a$, and denoted by $F_{a}$. To an analytic germ $F$, we make it correspond the ideal $I(F) \subset \mathcal{O}_{n}$ of germs of analytic functions which are zero on $F$.

We say $f\left(\in \mathscr{E}_{n}\right)$ is flat at 0 if $T f=0$.

\section{§3. Generalizations of $[4],[8]$}

The next lemma is similar to the lemma at p. 33 in $[7]$, and the method of the proof is the same.

Lemma 1. Let $f, g$ be in $\mathscr{E}_{n}\left(\right.$ resp. $\left.\mathcal{O}_{n}\right)$ and $a_{i}(x, t)(i=l+1, \ldots, n)$ germs at $0 \times[0,1]$ in $\mathbf{R}^{n} \times \mathbf{R}$ of $C^{\infty}$-functions (resp. analytic functions). Assume that

$$
\begin{aligned}
& \text { as germs at } 0 \times[0,1] f(x)-g(x)=\sum_{i=l+1}^{n} a_{i}(x, t)\left(\frac{\partial f}{\partial x_{i}} t+\frac{\partial g}{\partial x_{i}}(1-t)\right) \text {, } \\
& a_{i}(0, t)=0
\end{aligned}
$$

Then there exists $\tau \in \operatorname{Dif}_{n}\left(r e s p\right.$. Adif $\left._{n}\right)$ such that

$$
f \circ \tau=g
$$




$$
\tau(x)=\left(\tau_{1}(x), \ldots, \tau_{n}(x)\right)=\left(x_{1}, \ldots, x_{l}, \tau_{l+1}(x), \ldots, \tau_{n}(x)\right) .
$$

Proof. Let $X$ be a complete vector field on $\mathbf{R}^{n+1}$ such that the germ of $X$ at $0 \times[0,1]$ is $\frac{\partial}{\partial t}-\sum_{i=l+1}^{n} d_{i} \frac{\partial}{\partial x_{i}}$, and $\varphi_{t} 1$ parameter group of transformation defined by $X$. Then we have

$$
X_{\left(x, t^{\prime}\right)} F=\left.\frac{d F\left(\varphi_{t}\left(x, t^{\prime}\right)\right)}{d t}\right|_{t=0}
$$

The assumptions give

$$
\begin{aligned}
& X_{(x, t)} F=0, \text { near } 0 \times[0,1] \text { if } F=f t+g(1-t) \\
& \left.\varphi_{t}\left(0, t^{\prime}\right)=\left(0, t+t^{\prime}\right),\right] \quad \text { if } 0 \leqq t+t^{\prime} \leqq 1 \text { and } x \text { is near } 0 \\
& \left.\varphi_{t}\left(x, t^{\prime}\right)=\left(y, t+t^{\prime}\right)\right] .
\end{aligned}
$$

These show that $(f t+g(1-t)) \circ \varphi_{t}(x, 0)$ is a constant for any fixed $x$ near 0 , and the $\varphi_{1}(x, 0)$ is a local diffeomorphism around $x=0$. This gives the result.

As a corollary of Lemma 1 , we obtain the next lemma which is due to $[8]$.

Lemma 2. Let $f, g$ be in $\mathrm{m}^{2}\left(\mathscr{E}_{n}\right)$ (resp. $\mathrm{nt}^{2}\left(\mathcal{O}_{n}\right)$ such that $f-g$ is an element of the ideal generated by $\frac{\partial g}{\partial x_{i}} \frac{\partial g}{\partial x_{j}} x_{k}\left(\begin{array}{c}i, j=l+1, \ldots, n \\ k=1, \ldots, n\end{array}\right)$. Then there exists $\tau \in \operatorname{Dif}_{n}\left(r e s p\right.$. Adif $_{n}$ ) such that

$$
\begin{aligned}
& f \circ \tau=g, \\
& \tau(x)=\left(\tau_{1}(x), \ldots, \tau_{n}(x)\right)=\left(x_{1}, \ldots, x_{l}, \tau_{l+1}(x), \ldots, \tau_{n}(x)\right) .
\end{aligned}
$$

Proof. By the hypothesis, there exist $b_{i, j} \in \mathfrak{m}\left(\mathscr{E}_{n}\right)$ (resp. $\left.\mathfrak{m}\left(\mathcal{O}_{n}\right)\right)$ such that $f-g=\sum_{i, j=l+1}^{n} b_{i, j} \frac{\partial g}{\partial x_{i}} \frac{\partial g}{\partial x_{j}}$. We have

$$
\left.\frac{\partial(f-g)}{\partial x_{i}}=\sum_{j=l+1}^{n} c_{i, j} \frac{\partial g}{\partial x_{j}} \text {, where } c_{i, j} \text { is in } \mathfrak{m}\left(\mathscr{E}_{n}\right) \text { (resp. } \mathfrak{m}\left(\mathcal{O}_{n}\right)\right) .
$$

Set $\frac{\partial(f-g)}{\partial x_{i}}=\sum_{j=1+l}^{n} c_{i, j} \frac{\partial g}{\partial x_{j}}$ and $f-g=\sum_{i=l+1}^{n} d_{i} \frac{\partial g}{\partial x_{i}}$ where $d_{i}$ is in $\mathfrak{m}\left(\mathscr{E}_{n}\right)$ 
(resp. $\mathfrak{m}\left(\mathcal{O}_{n}\right)$ ) in the equation in Lemma 1. Then it is enough to solve the equations

$$
\begin{aligned}
& d_{i}=\sum_{j=l+1}^{n} a_{j} c_{i, j} t+a_{i}, l+1 \leqq i \leqq n, \\
& a_{i}(0, t)=0 .
\end{aligned}
$$

Let $A$ be the matrix whose $(i, j)$-component is $c_{i, j} t$ and $I$ the unit matrix. Then the equations above are

$$
\left(d_{i}\right)=(A+I)\left(a_{i}\right) \text { and } a_{i}(0, t)=0 .
$$

We easily see that $A+I$ has the inverse $B$ where components of $B$ are germs at $0 \times[0,1]$. This prove the lemma.

we use frequently the next lemma which is also shown in [8].

Lemma 3. Let $p$ be in $\mathscr{F}_{n}$ and $p(0)=0$. Then there exist an integer $k>0$ and $q_{1}, \ldots, q_{n} \in \mathscr{F}_{n}$ such that $p^{k}=\sum_{i=1}^{n} q_{i} \frac{\partial p}{\partial x_{i}}$.

The next lemma is the result about differentiable functions, see [3] for analytic functions.

Lemma 4. Let $g \in \mathscr{E}_{n}$ satisfy the following condition,

$$
\frac{\partial^{m} g}{\partial x_{n}^{m}}(0) \neq 0 \quad \text { for some } m>0 \text {. }
$$

Then there are $f$ in $\mathscr{E}_{n-1}\left[x_{n}\right]$ (polynomials in $x_{n}$ with coefficients in $\mathscr{E}\left(x_{1}\right.$, $\left.\ldots, x_{n-1}\right)$ ) and $\tau$ in $\operatorname{Dif}_{n}$ such that

$$
\begin{aligned}
& f \circ \tau=g, \\
& \tau(x)=\left(\tau_{1}(x), \ldots, \tau_{n}(x)\right)=\left(x_{1}, \ldots, x_{n-1}, \tau_{n}(x)\right) .
\end{aligned}
$$

Proof. The case $\frac{\partial g}{\partial x_{n}}(0) \neq 0$, this is trivial. Therefore, suppose $\frac{\partial g}{\partial x_{n}}(0)=0 . \quad$ Let 


$$
G=\left(\frac{\partial g}{\partial x_{n}}\right)^{2} x_{n}
$$

then for some $m^{\prime}>0$

$$
\frac{\partial^{m^{\prime}} G}{\partial x_{n}^{m^{\prime}}}(0) \neq 0
$$

Applying Malgrange's preparation theorem [6] to $G$ and $g$, we get

$$
Q \in \mathscr{E}_{n} R_{i} \in \mathscr{E}\left(x_{1}, \ldots, x_{n-1}\right) \quad(i=0, \ldots, p)
$$

such that

$$
g=Q G+\sum_{i=0}^{p} R_{i} x_{n}^{i}
$$

Take $f=\sum_{i=0}^{p} R_{i} x_{n}^{i}$, then

$$
g-f=Q G
$$

If we apply Lemma 2 to $f, g$, then there exists $\tau=\left(x_{1}, \ldots, x_{n-1}, \tau_{n}(x)\right) \in$ $\operatorname{Dif}_{n}$ such that $f \circ \tau=g$.

Theorem 1. (1) Let $G$ be in $\mathscr{E}_{n-1}\left[x_{n}\right]$ (resp. $\mathcal{O}_{n-1}\left[x_{n}\right]$ ). Suppose the discriminant of $G$ in $x_{n}$ is not flat at 0 (resp. not 0 ). Then there exist $F$ in $\mathscr{E}_{n-2}\left[x_{n-1}, x_{n}\right]$ (polynomials in $x_{n-1}, x_{n}$ with coefficients in $\mathscr{E}\left(x_{1}\right.$, $\left.\left.\ldots, x_{n-2}\right)\right)\left(\operatorname{resp} . \mathcal{O}_{n-2}\left[x_{n-1}, x_{n}\right]\right)$ and $\tau$ in $\operatorname{Dif}_{n}\left(\right.$ resp. Adif $\left.{ }_{n}\right)$ such that

$$
F \circ \tau=G
$$

(2) The statement in (1) remains valid for any $G \in \mathscr{E}_{n}\left(\right.$ resp. $\left.\mathcal{O}_{n}\right)$ which satisfies the following conditions;

$$
\begin{aligned}
& G=G_{1} \cdot G_{2} \text { for some } G_{1} \in \mathscr{E}\left(x_{1}, \ldots, x_{n-1}\right)\left(\text { resp. } \mathcal{O}\left(x_{1}, \ldots, x_{n-1}\right)\right) \\
& G_{2} \in \mathscr{E}_{n}^{\circ}\left(\operatorname{resp} . \mathcal{O}_{n}^{\circ}\right) \text {, such that }
\end{aligned}
$$

(i) for some $m>0 \quad \frac{\partial^{m} G_{2}}{\partial x_{n}^{m}}(0) \neq 0$,

(ii) $T G_{1} \neq 0$.

Proof. When $n=2$ and $G \in \mathcal{O}_{n}$ Part (2) of this theorem is proved in 
[4]. And when $G_{1}=1$ in Part (2) this theorem is shown in [8]. It is enough to prove the case $G \in \mathfrak{m}^{2}(\mathscr{E})\left(\right.$ resp. $\mathfrak{m}^{2}(\mathcal{O})$ ). If we apply Lemma 4 to $G_{2}$ in (2). Then there exist $f \in \mathscr{E}_{n-1}\left[x_{n}\right]$ and $\tau_{1} \in \mathrm{Dif}_{n}$ such that

$$
\begin{aligned}
& f \circ \tau_{1}=G_{2}, \\
& \tau_{1}=\left(x_{1}, \ldots, x_{n-1}, \tau_{n}(x)\right),
\end{aligned}
$$

and we get

$$
\left(G_{1} \cdot f\right) \circ \tau_{1}=\left(G_{1} \circ \tau_{1}\right) \cdot\left(f \circ \tau_{1}\right)=G_{1} \cdot G_{2}=G
$$

Here $G_{1} \cdot f$ satisfies the assumption of (1). So it is sufficient to prove (1). Let $S$ be the ring of quotients of $\mathscr{F}_{n-1}\left[x_{n}\right]$ with respect to the ideal of those elements which vanish at the origin. Then by Proposition III 4. 10 in [6] $\mathscr{F}_{n}$ is faithfully flat over $S$. Hence from Lemma 3 there exist an integer $k>0$ and $p_{1}, q_{1}, \ldots, q_{n} \in \mathscr{F}_{n-1}\left[x_{n}\right]$ such that $p T G^{k}=\sum_{i=1}^{n} q_{i} T \frac{\partial G}{\partial x_{i}}$ and $p(0) \neq 0$. Because of this, one sees that $T\left(\frac{\partial G}{\partial x_{i}}\right)^{2}(i=1, \ldots, n)$ have no common divisor $p$ (where $p$ is in $\mathscr{F}_{n-1}\left[x_{n}\right]$ and not in $\mathscr{F}\left(x_{1}, \ldots, x_{n-1}\right)$ ). Let $K$ be the quotient field over $\mathscr{F}\left(x_{1}, \ldots, x_{n-1}\right)$, and $K\left[x_{n}\right]$ the polynomial ring over $K$. Then by the fact that $K\left[x_{n}\right]$ is a principal ideal ring, it is shown that the ideal generated by $T\left(\frac{\partial G}{\partial x_{i}}\right)^{2} i=1, \ldots, n$ in $K\left[x_{n}\right]$ is $K\left[x_{n}\right]$. Hence there exist $\phi_{i} \in \mathscr{F}_{n-1}\left[x_{n}\right]$ such that

$$
\psi=\sum_{i=1}^{n} \phi_{i} \cdot T\left(\frac{\partial G}{\partial x_{i}}\right)^{2} \text { is not } 0 \text { and in } \mathscr{F}\left(x_{1}, \ldots, x_{n-1}\right) .
$$

By the theorem of $\mathrm{E}$. Borel, there exist $\Phi_{i} \in \mathscr{E}_{n-1}\left[x_{n}\right] i=1, \ldots, n$ such that $T \Phi_{i}=\phi_{i}$. And if we put

$$
\Psi=\sum_{i=1}^{n} \Phi_{i}\left(\frac{\partial G}{\partial x_{i}}\right)^{2}
$$

then $\Psi$ is in $\mathscr{E}\left(x_{1}, \ldots, x_{n-1}\right)$ and not flat at 0 . It is easily seen that there exist $\tau_{2} \in \operatorname{Dif}\left(x_{1}, \ldots, x_{n-1}\right)$ and $\Phi_{i, j}^{\prime} \in \mathscr{E}_{n-1}\left[x_{n}\right]$ such that

$$
\text { for some } m>0 \quad \frac{\partial^{m} \Psi \circ \tau_{2}}{\partial x_{n-1}^{m}}(0) \neq 0 \text {, }
$$




$$
\Psi \circ \tau_{2}=\sum_{i, j=1}^{n} \Phi_{i, j}^{\prime} \frac{\partial G \circ \tau_{2}}{\partial x_{i}} \frac{\partial G \circ \tau_{2}}{\partial x_{j}}
$$

Put $G \circ \tau_{2}=\sum_{i=0}^{l} f_{i} x_{n}^{i},\left(f_{i} \in \mathscr{E}\left(x_{1}, \ldots, x_{n-1}\right)\right)$ and apply Malgrange's preparation theorem to $\left(\Psi \circ \tau_{2}\right) \cdot x_{n-1}$ and $f_{i}$ then there exist $Q_{i} \in \mathscr{E}\left(x_{1}, \ldots, x_{n-1}\right), P_{i, j}$ $\in \mathscr{E}\left(x_{1}, \ldots, x_{n-2}\right)(i=0, \ldots, l j=0, \ldots, k)$ such that

$$
f_{i}=\left(\Psi \circ \tau_{2}\right) \cdot x_{n-1} \cdot Q_{i}+\sum_{j=0}^{k} P_{i, j} x_{n-1}^{j} \quad(i=0, \ldots, l) .
$$

If we put

$$
F=\sum_{i, j} P_{i, j} x_{n-1}^{j} x_{n}^{i}
$$

then $G \circ \tau_{2}-F$ is in the ideal generated by $\frac{\partial G \circ \tau_{2}}{\partial x_{i}} \frac{\partial G \circ \tau_{2}}{\partial x_{j}} x_{n-1}, i, j=1, \ldots, n$, and we can apply Lemma 2 to $G \circ \tau_{2}, F$. From this there exists $\tau \in \operatorname{Dif}_{n}$ such that $F \circ \tau=G$.

In the analytic case it is enough for the proof that we remind the fact that

(1) $\mathscr{F}_{n}$ is faithfully flat over $\mathcal{O}_{n}$

and (2) Weierstrass' preparation theorem.

Remark 1. Let $f$ be in $\mathscr{E}_{n}^{\circ}$ and let $g$ be in $\mathscr{E}_{n}$ and be flat at 0 . Then $f+g$ is in $\mathscr{E}_{n}^{\circ}$ and so $f+g$ can be transformed into a polynomial in two variables. On the contrary, $G+g$ such that $G$ takes the one form in Theorem 1 and $g$ flat at 0 cannot be necessarily transformed into a polynomial in two variables. For example $f=x^{2} y \pm e^{-1 / y^{2}}$ cannot be transformed not only into any polynomial but also into any analytic function (locally at 0 ). The reason is as follows. If $f$ can be transformed into some element of $\mathcal{O}_{2}$, then $\left|\frac{\partial f}{\partial x}\right|+\left|\frac{\partial f}{\partial y}\right|$ satisfies the inequality of Lojasiewicz (Theorem IV 4.1 in [6]) on some neighborhood of 0 in $\mathbf{R}^{2}$. However it is easily seen that $\left|\frac{\partial f}{\partial x}\right|+\left|\frac{\partial f}{\partial y}\right|$ does not satisfy the inequality on any neighborhood of 0 in $\mathbf{R}^{2}$. It is a contradiction.

If $f\left(\in \mathfrak{m}\left(\mathscr{E}_{n}\right)\right)$ can be transformed into an element of $\mathcal{O}_{n}$ (or a polynomial), then $f=\prod_{i=1}^{k} f_{i}^{i}$ for some elements $f_{i} \in \mathscr{E}_{n}$ where $\prod_{i=1}^{k} f_{i} \in \mathscr{E}_{n}^{\circ}$. 
The converse of this is not always true. For example, $f=\left(x^{2}+y^{2}\right)$. $\left(x+e^{-1 / z^{2}}\right)$ cannot be transformed into any element of $\mathcal{O}_{3}$. For the proof, see $[5]$.

But we have a partial converse

Theorem 2. For any $G \in \mathscr{E}_{2}\left(\right.$ resp. $\left.\mathcal{O}_{2}\right)$ such that $G=g_{1} \cdot g_{2}^{2}, g_{1} \cdot g_{2} \in$ $\mathscr{E}_{2}^{\circ}$ (resp. $\left.\mathcal{O}_{2}^{\circ}\right)$, there exists $\tau \in \mathrm{Dif}_{2}\left(\right.$ resp. Adif $\left.{ }_{2}\right)$ such that $G \circ \tau$ is a polynomial.

Proof. It is enough to prove the case $G \in \mathfrak{m}^{2}\left(\mathscr{E}_{2}\right)\left(\right.$ resp. $\mathfrak{m}^{2}\left(\mathcal{O}_{2}\right)$ ). Because of the hypothesis on $g_{1} \cdot g_{2}$, one sees that $g_{2}$ is in $\mathscr{E}_{2}^{\circ}$. Applying Theorem 1 to $g_{2}$, we see that $g_{2}$ is transformed into a polynimial, so we may assume that $g_{2}$ is a polynomial. From Lemma 3 it is easily seen that the greatest common measure of $T\left(\frac{\partial G}{\partial x}\right)^{2}$ and $T\left(\frac{\partial G}{\partial y}\right)^{2}$ is $g_{2}^{2}$. If $p, q\left(\in \mathscr{F}_{2}\right)$ have no common divisor, then the height of the ideal generated by $p, q$ is 2 , hence the ideal contains $x^{n}, y^{n}$ for some $n>0$. From this the ideal generated by $T\left(\frac{\partial G}{\partial x}\right)^{2}$ and $T\left(\frac{\partial G}{\partial y}\right)^{2}$ contains $x^{n} g_{2}^{2}, y^{n} g_{2}^{2}$ for some $n>0$. Consider a map $u: \mathscr{E}_{2} \rightarrow \mathscr{E}_{2}$ defined by

$$
u(f)=f \circ\left(\left(\frac{\partial G}{\partial x}\right)^{2} / g_{2}^{2},\left(\frac{\partial G}{\partial y}\right)^{2} / g_{2}^{2}\right)
$$

and $\hat{u}: \mathscr{F}_{2} \rightarrow \mathscr{F}_{2}$ defined by

$$
\hat{u}(f)=f \circ\left(T\left(\frac{\partial G}{\partial x}\right)^{2} / g_{2}^{2}, T\left(\frac{\partial G}{\partial y}\right)^{2} / g_{2}^{2}\right)
$$

If we use the terminology and Proposition III 1.6 in [6], then we see that $\hat{u}$ being quasi-finite, hence $u$ is guasi-finite, that is, for some $m^{\prime}>0$ the ideal $\mathfrak{p}$ generated by $\left(\frac{\partial G}{\partial x}\right)^{2}$ and $\left(\frac{\partial G}{\partial y}\right)^{2}$ contains $g_{2}^{2} \mathfrak{m}\left(\mathscr{E}_{2}\right)^{m^{\prime}}$. From this there exists a polynomial $f$ such that, $f g_{2}^{2}-g_{1} g_{2}^{2} \in \mathfrak{p m}\left(\mathscr{E}_{2}\right)$. Put $f g_{2}^{2}=F$ and apply Lemma 2 to $F, G$, then there exists $\tau \in \mathrm{Dif}_{2}$ such that $F \circ \tau=G$.

In the analytic case, we must remind that $\mathscr{F}_{n}$ is faithfully flat over $\mathcal{O}_{n}$. The proof is similar to that in the differentiable case. 


\section{§4. The Example of Whitney}

For any transcendental function $\nu$ (such that $\nu(0)=4)$, the germ of the function $f=x y(y-x)(y-(3+t) x)(y-\nu(t) x)$ at 0 in $\mathbf{R}^{3}$ cannot be transformed into any polynomial even through differentiable changes of coordinates.

\section{Proof. Suppose}

(i) $g$ is a polynomial in three variables with real coefficients,

(ii) $\tau$ is a diffeomorphism of $C^{\infty}$ class of a neighborhood $U$ of 0 in $\mathbf{R}^{3}$ onto a neighborhood of 0 in $\mathbb{R}^{3}$, where $\tau(0)=0$,

(iii) $g \circ \tau=f$ on $U$.

Let $V$ be the vanishing of $f$ on $U$, then $V$ consists of five analytic manifolds, hence the vanishing of $g$ on $\tau(U)$ (that is, $\tau(V)$ ) consits of five differentiable manifolds. It is shown in [6] (Proposition VI 3.11)

"Let $X_{0}$ be an analytic germ at 0 in $\mathbf{R}^{n}$ with $\operatorname{dim} X_{0}=k$. Suppose that $X_{0}$ contains the germ $V_{0}$ of a $C^{\infty}$ manifold of dimension $k$, then $V_{0}$ is the germ of an analytic manifold (which is then an irreducible component of $X_{0}$ )."

From this and the hypothesis that $g$ is a polynomial, one sees that each sheet of $\tau(V)$ is an analytic manifold. Let these sheets be $V_{1}, \ldots$, $V_{4}$. For each $i(=1, \ldots, 4)$ the set $\mathfrak{p}_{i}$ of those polynomials which vanish on $V_{i}$ is prime in the polynomial ring $\mathbf{R}\left[x_{1}, x_{2}, x_{3}\right]$ of 3 -variables over R. The reason is as follows. From the fact that the radical of $\mathfrak{p}_{i}$ is $\mathfrak{p}_{i}$ and that $\mathbf{R}\left[x_{1}, x_{2}, x_{3}\right]$ is noetherian, it is shown that $\mathfrak{p}_{i}=\overbrace{j=1}^{k_{i}} \mathfrak{q}_{i, j}\left(\mathfrak{q}_{i, j}\right.$ : prime) and the sum of the vanishing of $\mathfrak{q}_{i, j}\left(i=1, \ldots, k_{i}\right)$ is the vanishing of $\mathfrak{p}_{i}$, especially the sum of the vanishing of $\mathfrak{q}_{i, j}\left(j=1, \ldots, k_{i}\right)$ on $V_{i}$ is $V_{i}$, hence $k_{i}=1$ and $\mathfrak{p}_{i}=\mathfrak{q}_{i, 1}$ is prime. Let $W_{i}$ be the vanishing of $\mathfrak{p}_{i}$ in $\mathbf{C}^{3}$. Then one can see easily that $W_{i}$ is an algebraic variety of $\operatorname{dim} 2$ and that $\mathfrak{p}_{i} \mathbf{C}\left[x_{1}, x_{2}, x_{3}\right]$ is 1 in height. From this and the fact that $\mathbf{C}\left[x_{1}, x_{2}\right.$, $\left.x_{3}\right]$ is integral over $\mathbf{R}\left[x_{1}, x_{2}, x_{3}\right], \mathfrak{p}_{i}$ is a prime ideal of height 1 in $\mathbf{R}\left[x_{1}, x_{2}, x_{3}\right]$. Hence $\mathfrak{p}_{i}=p_{i} \mathbf{R}\left[x_{1}, x_{2}, x_{3}\right]$ for some $p_{i} \in \mathbf{R}\left[x_{1}, x_{2}, x_{3}\right]$, and the ideal of polynomials which vanish on $\tau(V)$ is $\prod_{i=i_{1}, \ldots, i_{j}} p_{i} \mathbf{R}\left[x_{1}, x_{2}\right.$, $x_{3}$ ] (not always $j=4$ ). For the remainder of this proof, we only have to 
proceed as in [9].

S. Izumi pointed out the following.

Remark 2. Using Artin's theorem in [1] we can easily prove the fact above, moreover Proposition VI 3.11 in [6] which we use in the proof above also can be shown by the same theorem.

\section{§5. Multiplication by Germs}

Let $f, g$ be in $\mathfrak{m}\left(\mathscr{E}_{n}\right)$ (resp. $\mathfrak{m}\left(\mathcal{O}_{n}\right)$ ), and $f^{-1}(0)=g^{-1}(0)$. Then here is a question whether there exists $\tau \in \operatorname{Dif}_{n}$ (resp. Adif ${ }_{n}$ ) such that $f=g \circ \tau$. This section give an answer to this question in the case that $f$ and $g$ have a special relation such that $f=\varphi g, \varphi(0)>0$.

$k$ in Lemma 3 is not always 1 . For example, $p=x^{6}+x^{4} y^{4}+y^{6}$ cannot have a solution of the equation

$$
p=\sum_{i=1}^{n} q_{i} \frac{\partial p}{\partial x_{i}}
$$

Definition. $\overline{\mathscr{E}}_{n}\left(\right.$ resp. $\left.\overline{\mathcal{O}}_{n}\right)$ is the set of germs $P \in \mathfrak{m}\left(\mathscr{E}_{n}\right)\left(\right.$ resp. $\left.\mathfrak{m}\left(\mathcal{O}_{n}\right)\right)$ which satisfy

$$
P=\sum_{i=1}^{n} q_{i} \frac{\partial P}{\partial x_{i}} \quad \text { for some } q_{i} \in \mathfrak{m}\left(\mathscr{E}_{n}\right)\left(\text { resp. } \mathfrak{m}\left(\mathcal{O}_{n}\right)\right)
$$

Proposition 1. For any $g \in \overline{\mathscr{E}}_{n}$ (resp. $\left.\overline{\mathcal{O}}_{n}\right), \phi \in \mathscr{E}_{n}$ (resp. $\mathcal{O}_{n}$ ) (such that $\phi(0)>0$ ), there exists $\tau \in \operatorname{Dif}_{n}$ ) (resp. Adif ${ }_{n}$ ) such that $\phi \cdot g=g \circ \tau$.

Proof. Let's proceed as in the proof of Lemma 2. It is enough to have germs $a_{i}(x, t)$ which satisfy the following conditions;

$$
\begin{gathered}
a_{i}(0, t)=0 \\
(\phi-1) g=\sum_{i=1}^{n} a_{i}(x, t)\left(\frac{\partial(\phi-1) g}{\partial x_{i}} t+\frac{\partial g}{\partial x_{i}}\right)
\end{gathered}
$$

By the hypothesis, there exist $b_{j} \in \mathfrak{m}\left(\mathscr{E}_{n}\right)\left(\right.$ resp. $\left.\mathfrak{m}\left(\mathcal{O}_{n}\right)\right)$ such that 


$$
g=\sum_{j=1}^{n} b_{j} \frac{\partial g}{\partial x_{j}}
$$

From this, (2) becomes

$$
\sum_{j}(\phi-1) b_{j} \frac{\partial g}{\partial x_{j}}=\sum_{i, j} a_{i}(x, t) b_{j} \frac{\partial \phi}{\partial x_{i}} \cdot \frac{\partial g}{\partial x_{j}} t+\sum_{i} a_{i}(x, t) \frac{\partial g}{\partial x_{i}}\{(\phi-1) t+1\}
$$

If we get the following relation for all $i$,

$$
(\phi-1) b_{i}=\sum_{j} a_{j}(x, t) b_{i} \frac{\partial \phi}{\partial x_{j}} t+a_{i}(x, t)\{(\phi-1) t+1\}
$$

then (3) is established. Hence it is sufficient to find $a_{i}(x, t)$ which satisfy (1) and (4). Let $A$ be the matrix whose $(i, j)$-component is $b_{i} \frac{\partial \phi}{\partial x_{j}} t$ and $I$ the unit matrix. Then (4) is equal to

$$
\left(\begin{array}{c}
(\phi-1) b_{1} \\
\vdots \\
(\phi-1) b_{n}
\end{array}\right)=(A+\{(\phi-1) t+1\} I)\left(\begin{array}{c}
a_{1} \\
\vdots \\
a_{n}
\end{array}\right) .
$$

From $b_{i} \in \mathfrak{m}\left(\mathscr{E}_{n}\right)\left(\right.$ resp. $\left.\mathfrak{m}\left(\mathscr{E}_{n}\right)\right)$, the determinant of $A+\{(\phi-1) t+1\} I$ does not vanish at any point of $0 \times[0,1]$, hence there is the inverse matrix $B$ of $A+\{(\phi-1) t+1\} I$ (where all components of $B$ are germs at $0 \times[0,1]$ of $C^{\infty}$-functions (resp. analytic ones)). By $b_{j}(0)=0$, all components of $B\left(\begin{array}{c}(\phi-1) b_{1} \\ \vdots \\ (\phi-1) b_{n}\end{array}\right)$ (that is, $\left.a_{i}\right)$ satisfy (1). The proposition follows.

The following is the converse of this result in the analytic case.

Proposition 2. Suppose $g \in \mathrm{m}\left(\mathcal{O}_{n}\right)$ satisfies the following condition; for any $t \in[1,2]$, there exists $\tau_{t} \in$ Adif $_{n}$ such that $t g=g \circ \tau_{t}$. Then $g \in$ $\overline{\mathcal{O}}_{n}$.

Proof. For each integer $k(>1), T_{k}^{n}$ denotes the natural map from $\left(\mathcal{O}_{n}\right)^{n}$ to $\left(\mathcal{O}_{n}\right)^{n} / \mathfrak{u t}^{k}\left(\mathcal{O}_{n}\right) \times\left(\mathcal{O}_{n}\right)^{n},\left(\mathcal{O}_{n}\right)^{n} / \mathrm{m}^{k}\left(\mathcal{O}_{n}\right) \times\left(\mathcal{O}_{n}\right)^{n}$ is a vector space of finite dimension over $\mathbf{R}$. Regard Adif $_{n}$ as contained in $\left(\mathcal{O}_{n}\right)^{n}$, then the set of $T_{k}^{n} \tau_{t}(t \in[1,2])$ is contained in a finite dimensional vector space over R. Hence there exist $t_{m} \in[1,2](m=1,2, \ldots)$ such that $t_{m}$ and $T_{k}^{n} \tau_{t_{m}}$ 
converge. From these convergence one sees that when $m \rightarrow \infty, m^{\prime} \rightarrow \infty$ then $\frac{t_{m}}{t_{m^{\prime}}}$ and $T_{k}^{n} \tau_{t_{m}} \circ \tau_{t_{m}}^{-1}$ converge respectively to 1 and $T_{k}^{n} I$ (where $I$ is the identity map). Hence from the beginning we may assume that $t_{m}$, $T_{k}^{n} \tau_{t_{m}}$ converge respectively to 1 and $T_{k}^{n} I$ (when $m \rightarrow \infty$ ), and that

$$
g \circ \tau_{t_{m}}=t_{m} g
$$

For sufficiently large $m$, and $1 \leqq t \leqq t_{m}, \frac{t_{m}-t}{t_{m}-1} I+\frac{t-1}{t_{m}-1} \tau_{t_{m}}$ belongs to $\operatorname{Adif}_{n}$. Let $f_{m}(x, t)=g \circ\left(\frac{t_{m}-t}{t_{m}-1} I+\frac{t-1}{t_{m}-1} \tau_{t_{m}}\right)$. Then $f_{m}(x, t)$ is a germ at $0 \times\left[1, t_{m}\right]$ in $\mathbf{R}^{n} \times \mathbf{R}$ of analytic function, and satisfies

$$
\begin{aligned}
& f_{m}(x, 1)=g, \\
& f_{m}\left(x, t_{m}\right)=t_{m} g .
\end{aligned}
$$

We have that

$$
\begin{aligned}
& \frac{f_{m}\left(x, t_{m}\right)-f_{m}(x, 1)}{t_{m}-1}-\frac{\partial f_{m}(x, 1)}{\partial t} \text { converges to } 0 \text { in } \\
& \left.\frac{\mathcal{O}_{n}}{\mathfrak{m}^{k}\left(\mathcal{O}_{n}\right)} \text { (when } m \rightarrow \infty\right) .
\end{aligned}
$$

This is because letting $f_{m}(x, t)=\sum_{l=0}^{\infty} h_{m, l}(x)(t-1)^{l}$ we have

$$
\begin{array}{r}
h_{m, l}(x) \in \mathfrak{m}^{l}\left(\mathcal{O}_{n}\right) \text {, and } h_{m, l}(x) \text { converges in } \mathcal{O}_{n} / \mathfrak{m}^{k}\left(\mathcal{O}_{n}\right) \\
\text { (when } m \rightarrow \infty) .
\end{array}
$$

From this we have

$$
f_{m}(x, t)=\sum_{l=0}^{k-1} h_{m, l}(x)(t-1)^{l} \quad \bmod \mathfrak{m}^{k}\left(\mathcal{O}_{n}\right)
$$

From the equations

$$
\begin{gathered}
\frac{f_{m}\left(x, t_{m}\right)-f_{m}(x, 1)}{t_{m}-1}=\frac{t_{m} g-g}{t_{m}-1}=g, \\
\frac{\partial f_{m}}{\partial t}(x, 1)=\left.\sum_{i=1}^{n} \frac{\partial g}{\partial x_{i}} \frac{\partial\left(\frac{t_{m}-t}{t_{m}} I+\frac{t}{t_{m}} \tau_{t_{m}}\right)_{i}}{\partial t}\right|_{t=1},
\end{gathered}
$$


and the fact that $T_{k} \mathfrak{p}$ where $\mathfrak{p}$ is the ideal generated by $\frac{\partial g}{\partial x_{i}} x_{j}$ is a linear subspace of a finite dimensional vector space over $\mathbf{R}$, we see that $g$ is contained in $\mathfrak{p}+\mathfrak{m}^{k}\left(\mathcal{O}_{n}\right)$. Because $k$ is arbitrary, and by Theorem of Krull we see that $g$ is centained in $\mathfrak{p}$.

Remark 3. In the same way as in the above proofs, we can prove that for any $f \in \mathfrak{m}\left(\mathscr{E}_{n}\right)$, the following two conditions are equivalent;

(1) for any $\phi \in \mathscr{E}_{n}$ (such that $\phi(0)>0$ ) there exists $\tau \in \operatorname{Dif}_{n}$ such that $T(f \circ \phi)=T(f \circ \tau)$,

(2) there exists $q_{i} \in \mathscr{F}_{n}(i=1, \ldots, n)$ such that $q_{i}(0)=0 \quad T f=\sum_{i=1}^{n} q_{i} T$ $\frac{\partial f}{\partial x_{i}}$.

\section{§6. A Sufficient Condition}

In this section we consider only the case $n=2$.

Lemma 5. Let $f_{i}(i=1, \ldots, n)$ be in $\mathscr{E}_{2}$ such that $g=\prod_{i=1}^{n} f_{i}$ is in $\mathcal{O}_{2}^{\circ}$. Then there exist $\phi_{i} \in \mathscr{E}_{2}(i=1, \ldots, n)$ such that each $\phi_{i} f_{i}$ is analytic and $\prod_{i=1}^{n} \phi_{i}=1, \phi_{i}(0)=1$.

Proof. Here we don't distinguish a function from the germ defined by its function.

We have the following facts

(i) $\mathcal{O}_{n}$ and $\mathscr{F}_{n}$ are unique factorization rings,

(ii) for any prime ideal $\mathfrak{p}$ of $\mathcal{O}_{n}, \mathfrak{p} \mathscr{F}_{n}$ is prime in $\mathscr{F}_{n}$ (Zariski-Nagata). By these it is easily seen that we can assume $T f_{i}$ are prime in $\mathscr{F}_{2}$, and that there exist $p_{i} \in \mathscr{F}_{2}(i=1, \ldots, n)$ where $p_{i}(0)=1 \prod_{i=1}^{n} p_{i}=1 \quad p_{i} T f_{i}=q_{i}$ $\in \mathcal{O}_{2}$. Let $\psi_{i}(i=1, \ldots, n)$ be in $\mathscr{E}_{2}$ such that $T \psi_{i}=p_{i}(i=1, \ldots, n)$. Then we see that $q_{i}-\psi_{i} f_{i}$ are flat at 0 .

For each $i$, the vanishing of $f_{i}$ is the vanishing $V_{i}$ of $q_{i}$. The reason is as follows. Any analytic germ $X$ in $\mathbf{R}^{2}$ is described in the following form (by some linear transformation) (see p.57 in [6]);

" $p(x, y)$ is a distinguished polynomial in $x$ with coefficients in $\mathcal{O}(y)$ and $X$ is the vanishing of $p$. " 
Hence $V_{i}$ is $\{0\}$ or a sum of curves. If $V_{i}$ is a sum of curves and $f_{j}$ vanishes on a subgerm $(\neq\{0\})$ of $V_{i}(j \neq i)$, then there is a sequence $a_{n}$ $(\rightarrow 0)$ in $V_{i}$ such that $f_{j}\left(a_{n}\right)=0(n=1,2 \ldots)$. By the fact that $q_{j}-\psi_{j} f_{j}$ is flat at 0 , for any $N>0$ there is a neighborhood of 0 where we have

$$
\left|q_{j}(x)-\phi_{j} f_{j}(x)\right| \leqq|x|^{N}
$$

From these we see that for sufficient large $n$

$$
\left|q_{j}\left(a_{n}\right)\right| \leqq\left|a_{n}\right|^{N}
$$

Now, from the hypothesis that $\Pi q_{j}=g$ is in $\mathcal{O}_{2}^{\circ}$, one sees easily that the ideal (generated by $q_{i}, q_{j}$ ) is 2 in height, and that the ideal is contained in $I\left(V_{i} \cap V_{j}\right)$. Hence $I\left(V_{i} \cap V_{j}\right)$ is 2 in height, and $V_{i} \cap V_{j}=\{0\}$. It is shown in [6] (Corollary IV 4.4) that any two analytic sets $X, Y$ are regularly situated (i.e. locally there exists a pair of constants $c>0$ and $\alpha>0$ such that for every $x$ in $\left.X d(x, Y) \geqq c d(x, X \cap Y)^{\alpha}\right)$. From this there exists a pair of $c>0$ and $\alpha>0$ such that for sufficiently large $n$

$$
d\left(a_{n}, V_{j}\right) \geqq c\left|a_{n}\right|^{\alpha}
$$

But, from the inequality of Lojasiewicz, we have locally for some constants $c^{\prime}>0, \alpha^{\prime}>0$

$$
\left|q_{j}(x)\right| \geqq c^{\prime}\left(d\left(x, V_{j}\right)\right)^{\alpha^{\prime}}
$$

especially for sufficiently large $n$

$$
\left|q_{j}\left(a_{n}\right)\right| \geqq c^{\prime}\left(d\left(a_{n}, V_{j}\right)\right)^{\alpha^{\prime}} .
$$

From (1), (2), (3), we get for sufficiently large $n$

$$
c^{\prime}\left(d\left(a_{n}, V_{j}\right)\right)^{\alpha^{\prime}} \leqq\left(\frac{d\left(a_{n}, V_{j}\right)}{c}\right)^{\frac{N}{\alpha}} .
$$

By the fact that $N$ is arbitrary and $d\left(a_{n}, V_{j}\right)$ is not 0 , this is a contradiction. Thus we have shown that

$$
V_{i} \text { is }\{0\},
$$

or that 
$f_{j}$ does not vanish on any point $(\neq 0)$ of $V_{i}(j \neq i)$.

From the hypothesis $\left(\Pi f_{i}=g\right.$ ), the sum of the vanishing (of $f_{i}$ on $V_{i}$ ) is $V_{i}$. Hence one sees that $V_{i}$ is the vanishing of $f_{i}$.

On the other hand, any analytic set $X$ in $\Omega$ (open in $\mathbf{R}^{2}$ ) is coherent (i.e. for any $a \in \Omega$ and for any finite system $\left\{h_{i}\right\}$ of generators of $I\left(X_{a}\right)$, there exists a neighborhood $\Omega^{\prime}$ of $a$ such that for any $b \in \Omega^{\prime} h_{i}$ generate $\left.I\left(X_{b}\right)\right)$. The reason is, if $I\left(X_{a}\right)$ is 2 in height, then the above statement is trivial, if $I\left(X_{a}\right)$ is 1 in height, then $I\left(X_{a}\right)$ is principal and is generated by $h$ (where $h$ is in $\mathcal{O}_{2}^{\circ}$ ). From this we have near $a$

$$
\left|\frac{\partial h}{\partial x}(b)\right|+\left|\frac{\partial h}{\partial y}(b)\right| \neq 0, b \neq a
$$

and one sees easily that near $a I\left(X_{b}\right)$ is generated by $h$.

In [6] Malgrange has shown the following theorem (VI 3.10): Let $K\left(X_{0}\right)$ be the ideal in $\mathscr{E}_{n}$ of $C^{\infty}$ functions vanishing on $X_{0}$, then the following properties are equivalent,

(a) $K\left(X_{0}\right)=I\left(X_{0}\right) \mathscr{E}_{n}$,

(b) $X_{0}$ is coherent at 0 .

From this, if $V_{i} \neq\{0\}$ then there exists $\phi_{i}^{\prime} \in \mathscr{E}_{2}$ such that $f_{i}=q_{i} \phi_{i}^{\prime}$.

Malgrange [6] also has proved the fact (Theorem VI 1.1'): Let $\Omega$ be an open set in $\mathbf{R}^{n}$ and $h_{1}, \ldots, h_{p}$ analytic functions in $\Omega$. Let $\phi \in \mathscr{E}(\Omega)$ (the set of $C^{\infty}$ functions defined on $\Omega$ ). Then $\phi$ can be written in the form $\phi=\sum_{i=1}^{p} h_{i} \psi_{i}$ (with $\psi_{i} \in \mathscr{E}(\Omega)$ ) if and only if for any $a \in \Omega$, the Taylor expansion $T_{a} \phi$ of $\phi$ at $a$ belongs to the ideal generated by $T_{a} h_{i}$ in $\mathscr{F}_{n}$. From this, if $V_{i}=0$ then there exists $\phi_{i}^{\prime} \in \mathscr{E}_{2}$ such that $f_{i}=q_{i} \phi_{i}^{\prime}$. Since $\Pi f_{i}=\Pi q_{i}, p_{i}(0)=1$. Hence $\Pi \phi_{i}^{\prime}=1$ and $\phi_{i}^{\prime}(0)=1$. Thus the lemma is proved.

Remark 4. In the lemma above we see that an analytic set in $\mathbf{R}^{2}$ is coherent. But the one in $\mathbf{R}^{3}$ is not always coherent. The counter example is the "umbrella" (p. 95 in [6]).

Lemma 6. Let $f$ be such that $f=\prod_{i=1}^{k} f_{i}^{i}$ (where $\left.\prod_{i=1}^{k} f_{i} \in \mathscr{E}_{2}^{\circ}\right)$. Then there exist $\tau \in \operatorname{Dif}_{2}$ and $\phi \in \mathscr{E}_{2}-\mathfrak{m}\left(\mathscr{E}_{2}\right)$ such that $\phi(f \circ \tau)$ is analytic. 
Proof. Applying Theorem 1 to $g=\prod_{i=1}^{k} f_{i}$, we get $\tau \in \mathrm{Dif}_{2}$ such that $g \circ \tau$ is analytic. From Lemma 5 there exist $\phi_{i} \in \mathscr{E}_{2}(i=1, \ldots, k)$ such that $\phi_{i}\left(f_{i} \circ \tau\right)$ are analytic and $\prod_{i=1}^{k} \phi_{i}=1, \phi_{i}(0)=1$. Hence $\prod_{i=1}^{k}\left\{\phi_{i}^{i} \cdot\left(f_{i} \circ \tau\right)^{i}\right\}$ is analytic and equal to $\left(\prod_{i=1}^{k} \phi_{i}^{i}\right)(f \circ \tau)$.

Combining this and Proposition 1, we have

Theorem 3. For any $f \in \overline{\mathscr{E}}_{2}$ such that $f=\prod_{i=1}^{k} f_{i}^{i}, \prod_{i=1}^{k} f \in \mathscr{E}_{2}^{\circ}$ there exist $\tau \in \mathrm{Dif}_{2}$ such that $f \circ \tau$ is analytic.

Remark 5. Let $f \in \mathscr{E}_{2}$ be such that for some $q_{i} \in \mathfrak{m}\left(\mathscr{F}_{n}\right), T f=\sum_{i=1}^{n}$ $q_{i} T \frac{\partial f}{\partial x_{i}}$, then by Remark 3 there exist $\tau \in \operatorname{Dif}_{2}$ and $\psi \in \mathscr{E}_{2}$ such that $\psi$ is flat at 0 and $f \circ \tau+\phi$ is analytic.

\section{§7. Appendix: A Proof to Cerf's Result}

Let us recall the following concept introduced by Cerf [2].

Let $W$ be a $C^{\infty}$-manifold, $f$ a $C^{\infty}$-function defined on $W$, and $c$ a critical point of $f$. One calls condimension of critical point $c$ the codimension of the ideal generated by the germs of the first partial derivatives of $f$ at $c$ in the ring of germs of $C^{\infty}$-function: $W \rightarrow \mathbf{R}$ zero at $c$.

Critical points of codimension 0,1 , or 2 are called respectively points critiques du type de Morse, points de naissance, and points critiques du type queue d'aronde.

We present a proof to the following Cerf's result (shown at p. 23 in $[2])$.

Proposition 3. If $f \in \mathfrak{m}\left(\mathscr{E}_{n}\right)$ has a critical point of codimension 1 (resp. 2) at 0 , then there exists $\tau \in \mathrm{Dif}_{n}$ such that

$$
\begin{gathered}
f \circ \tau=-x_{1}^{2}-\cdots-x_{i}^{2}+x_{i+1}^{2}+\cdots+x_{n-1}^{2}+x_{n}^{3}, \\
\left(\text { resp. }=-x_{1}^{2}-\cdots-x_{i}^{2}+x_{i+1}^{2}+\cdots+x_{n-1}^{2} \pm x_{n}^{4}\right) .
\end{gathered}
$$

Proof. The proof of the case of codim 2 follows in the same way as its of codim 1 , so we prove only the case of codim 1 . 
Let $\mathfrak{p}(f)$ be the ideal generated by the germs of the first partial derivatives of $f$ in $\mathscr{E}_{n}$. We have

$$
\mathrm{m} / \mathrm{p}=\mathbf{R}^{1}, \quad \bmod m^{2}
$$

Hence, after some linear transformation, we can assume

$$
\begin{aligned}
& \frac{\partial f}{\partial x_{i}} \in \mathfrak{m}-\mathrm{m}^{2}, \quad i=1, \ldots, n-1, \\
& \frac{\partial f}{\partial x_{n}} \in \mathfrak{m}^{2}-\mathfrak{m}^{3} .
\end{aligned}
$$

Therefore there exist $g_{0}, g_{1}$ and $g_{2} \in \mathscr{E}\left(x_{1}, \ldots, x_{n-1}\right)$ and $h \in \mathscr{E}_{n}-\mathfrak{m}$ such that

$$
\begin{aligned}
& f=g_{0}+g_{1} x_{n}+g_{2} x_{n}^{2}+h x_{n}^{3}, \\
& g_{0}: \text { of Morse type, } \\
& g_{1} \in \mathfrak{M}^{2}\left(\mathscr{E}_{n-1}\right), \\
& g_{2} \in \mathfrak{M}\left(\mathscr{E}_{n-1}\right) .
\end{aligned}
$$

Applying Morse's theorem to $g_{0}$, we have

$$
g_{0}=-x_{1}^{2}-\cdots-x_{i}^{2}+x_{i+1}^{2}+\cdots+x_{n-1}^{2} .
$$

Let $g_{2}=\sum_{i=1}^{n-1} a_{i} x_{i}+g_{3}$ such that $a_{i}$ are constants and $g_{3} \in \mathfrak{m}^{2}$. Then, transforming $x_{i}$ by $x_{i} \pm \frac{a_{i}}{2} x_{n}^{2}$, we only have to prove the case $g_{2} \in \mathrm{m}^{2}$. Let us apply Lemma 2 to $f$ and $G=g_{0}+h x_{n}^{3}$. We see easily that $\mathfrak{p}(G)$ is generated by $x_{1}, \ldots, x_{n-1}$, and $x_{n}^{2}$, and that $\mathfrak{p}^{2}(G) \mathfrak{m}$ contains $g_{1} x_{1}+g_{0} x_{n}^{2}$. From Lemma 2 there exists $\tau \in \operatorname{Dif}_{n}$ such that $f \circ \tau=G$. Let $\tau^{\prime} \in \operatorname{Dif}_{n}$ denote $\left(x_{1}, \ldots, x_{n-1}, h^{1 / 3} x_{n}\right)$. Then $f \circ \tau \circ \tau^{\prime}=-x_{1}^{2}-\cdots-x_{i}^{2}+x_{i+1}^{2}+\cdots+$ $x_{n-1}^{2}+x_{n}^{3}$.

\section{References}

[1] Artin, M., On the solution of analytic equations, Inventions Math., 5 (1968), 277291.

[2] Cerf, J., La stratification naturelle des espaces de fonctions différentiables réel- 
les et le théoréme de la pseudoisotopie, Publ. Math. I.H.E.S., 39 (1970).

[3] Levinson, N., A canonical form for an analytic function of several variables at a critical point, Bull. Amer. Math. Soc., 66 (1960) 68-69.

[4] Levinson, N., A polynomial canonical form for certain analytic functions of two variables at a critical point, Bull. Amer. Math. Soc., 66 (1960) 366-368.

[5] Lojasiewicz, S., Ensembles semi-analytiques, Cours Faculté des Sciences d'Orsay Momeographié I.H.E.S., Buressur-Yvette, July, 1965.

[6] Malgrange, B., Ideals of differentiable functions, Oxford University Press, 1966.

[7] Mather, J., Stability of $C^{\infty}$ mappings; III Finitely determined map germs, Publ. Math. I.H.E.S., 35 (1968), 127-156.

[8] Tougeron, J. Cl., Idéaux de fonctions différentiable, 1. Ann. Inst. Fourier, 18, 1 (1968) $177-240$.

[9] Whitney, H., Local properties of analytic varieties, M. Morse Jubilee Volume, Differential anp Combinatorial Topology, Princeton Univ. Press, 1965, p. 205-244.

Department of Mathematics, Kyoto University. 